\title{
GLOTTOPOLITIQUE(S) ET POUVOIR(S) : TEXTES ET CONTEXTES
}

BENMESSAOUD, Redha ${ }^{1}$

RÉSUMÉ : Le présent article revisite la notion de glottopolitique dans une perspective synchronique visant des périodes précises de l'histoire européenne : des années 1920 aux années 1970. Il y a, d'un côté, les textes, au sens législatif du terme, c'est-à-dire les politiques linguistiques et, de l'autre, les contextes, au sens spatial du terme, autrement dit, les pays. Textes et contextes sont donc les maîtres mots de cet article qui se veut un regard neutre sur les politiques linguistiques, appliquées par les pouvoirs en place, et leurs incidences sur les langues et leurs usages. Si ces politiques sont tout à fait nécessaires, pour la gestion des langues, et, par conséquent, compréhensibles, à bien des égards, leurs finalités, elles, sont souvent peu avouables.

MOTS-CLÉS : Langue ; pouvoir ; politiques linguistiques (textes) ; Europe (contexte) ; autarcie linguistique ; superstructure.

\section{GLOTTOPOLITICS AND POWERS: TEXTS AND CONTEXTS}

ABSTRACT: This article will synchronically revisit the notion of glottopolitics during specific periods of European history, mainly those going from the 1920s to the 1970s. It will, herein, consider two major keywords: texts in a legislative meaning (i.e., the linguistic policies), and contexts in a spatial meaning (i.e., countries). This article, as it were, bears impartiality over the linguistic policies as applied by the powers in place, and their impact on languages and their usages. Now, if these policies are absolutely necessary for the management of languages, and thus intelligible, yet, their purposes remain, in many respects, unrecognizable.

KEYWORDS: Language; power; linguistic policies (texts); Europe (context); linguistic autarky; superstructure.

\section{INTRODUCTION}

Le rapport langue-politique peut être appréhendé selon plusieurs paramètres dans la mesure où il met en jeu le pouvoir en place, le peuple et la langue en tant que moyen de communication,

\footnotetext{
${ }^{1}$ Maître de conférences, Université Frères Mentouri Constantine 1, Algérie. E-mail : redhabenmessaoud @ yahoo.fr Jangada | ano 9, nr. 17, jan/jun, 2021 | ISSN 2317-4722 


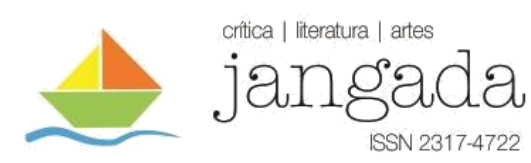

susceptible d'être utilisé à des fins politiques. Il y est, en effet, question de la langue en tant qu'outil au service de la politique du pouvoir et des politiques linguistiques adoptées par ce dernier pour gérer les situations plurilingues qui sont parfois conflictuelles. Par quelque bout qu'on la prenne, la langue relève inéluctablement de la politique et les dirigeants, aussi différents soient-ils, en ont toujours eu conscience. "[...] Les dictateurs, de César à Staline, se sont préoccupés de la langue, y reconnaissant l'image la plus fidèle d'un pouvoir nu, qui n'a même pas à dire son nom" (MILNER, 1978, p. 28).

La langue est toujours le reflet de la politique suivie par le pouvoir en place qui excelle dans la manipulation des masses, et ce, dans un but souvent peu avouable.

Les pratiques linguistiques ne sont jamais le pur produit de leurs propres développements, mais celui de politiques linguistiques qui sont souvent dépendantes de changements politiques, économiques et sociaux. La formation des États-nations est souvent un élément déterminant dans l'élaboration de ces politiques [...] (GOUJON, 2004, p. 59)

L'ombre du pouvoir est toujours présente dans le linguistique. La langue est sous son emprise et il en fait ce que bon lui semble. De la manipulation des foules jusqu'à l'assujettissement de tout un peuple, la langue n'est jamais une fin en soi. C'est un outil aux mains du pouvoir qui se trouve être l'enjeu de tout problème linguistique.

C'est dans cette perspective glottopolitique que s'inscrit donc le présent article, basé sur une réflexion théorique. Il vise à montrer, à l'aide d'exemples concrets, que la politique et la langue ont toujours été indissociables dans tous les régimes connus à travers le monde : démocratique, autocratique, monarchique ou fédéral. Ces exemples sont en fait des études de cas variés : de la Turquie d'Atatürk à la Grèce des katharévoussiani.

\section{ATATÜRK : LA LANGUE AU SERVICE DU CHANGEMENT POLITIQUE OU LA LANGUE COMME SUPERSTRUCTURE}

Considéré comme l'un des grands réformateurs du $\mathrm{XX}^{\mathrm{e}}$ siècle, Mustafa Kemal Atatürk, de formation militaire, accède au pouvoir en 1923 et impose d'emblée sa vision d'une Turquie moderne, républicaine et laïque. Ainsi : 


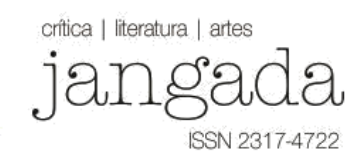

[...] dès les premières années de la République, une action rénovatrice a été engagée dans tous les domaines, et a été poursuivie sans relâche sous l'inspiration des principes kémalistes dont l'objectif majeur était de moderniser la vie sociale, de laïciser l'État, d'orienter le peuple vers des modes de pensée moderne [...] (VARDAR, 1981, p. 18)

Des mesures en faveur des femmes sont prises et une rupture totale avec le régime précédent est déclarée surtout en ce qui concerne la langue turque : plus d'alphabet arabe pour cette langue. Le 10 août 1928, soit cinq ans après avoir pris les rênes du pouvoir, Atatürk disait ceci :

Camarades, notre belle langue, riche et harmonieuse, montrera sa valeur grâce au nouvel alphabet turc. Nous sommes obligés de nous affranchir de ce cercle de fer qui entourait notre tête depuis des siècles. Nous ne voulons plus de ces signes incompréhensibles. Nous voulons comprendre notre langue. Avec le nouvel alphabet, nous arriverons à la comprendre d'une façon parfaite, j'en suis sûr. Soyez-le aussi. Citoyens, apprenez vite les nouveaux caractères turcs. Enseignez-les au villageois, au pâtre, au portefaix, au batelier et à toutes les couches de la nation. Songez qu'il est honteux pour une nation d'avoir dans son sein $10 \%$ seulement de personnes sachant lire et écrire et $90 \%$ d'illettrés. Beaucoup de progrès ont été réalisés. Cependant la chose que nous devons faire aujourd'hui, et qui n'est certes pas la dernière, est comme je l'ai dit, nécessaire. Sachez que c'est un devoir national et patriotique. Dans un an ou deux ans au plus tard, tout le corps social turc apprendra le nouvel alphabet. Notre nation prouvera que son écriture et son intelligence sont au niveau de celles du monde civilisé. (Cité dans AKINCI, 2005)

Désormais, le turc doit s'écrire en caractères latins. Selon Berke Vardar (1981, p. 19), cette décision, justifiée de surcroît, répondait en fait à deux impératifs : le premier est extralinguistique, il s'agit du changement politique entraînant inévitablement un changement au niveau de la langue ; le second est linguistique ou plus exactement phonologique à partir du moment où le nombre de voyelles, utilisées jusque-là, n'était pas adéquat avec le système phonologique turc. Le changement n'a pas touché uniquement l'alphabet, il s'est étendu ensuite et progressivement aux mots d'origine arabe et persane. Mais l'auteur, cité plus haut, ne dit pas 


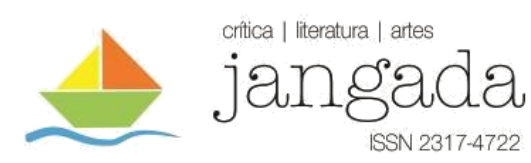

tout concernant cette décision de modifier certaines structures de la langue. Il y avait certes cette volonté de ce nouveau président de tout nationaliser, de rompre en quelque sorte avec l'Empire ottoman, mais il n'en demeure pas moins qu'il y avait un autre dessein, celui de mettre un terme à l'influence de l'islam par le biais justement du persan et de l'arabe. D'ailleurs, un de ses compatriotes, Genç Osman, l'affirme en ces termes :

[...]Atatürk et les kémalistes ont tout mis en œuvre pour supprimer l'influence de l'islam dans la société turque. Tout au long des différents articles, il a été démontré comment le Califat a été détruit, comment des hodjas par milliers ont été pendus et emprisonnés, comment des milliers de madrasas ont été fermés, comment l'enseignement du Saint Coran a été interdit... Les exemples sont fleuves et refuser de le voir reviendrait à nier l'évidence. (2014)

Le comble dans tout cela, c'est que, sur ordre d'Atatürk, même l'appel à la prière, fait d'habitude en arabe, a été traduit en turc, mais, aussi anecdotique que cela puisse paraître, un seul mot a été épargné par les traducteurs. Selon Genç (2014), il s'agit de "felâh" qui signifie en français "félicité".

L'équivalent en turc existe bien : "kurtulus" mais le seul bémol, en plus du sens précédent, c'est qu'il signifie aussi “libération”. Pour les Kémalistes, il n'était pas question que la libération vienne de la mosquée. C'est pour cette raison qu'ils ont préféré garder le mot arabe. Mais cela s'inscrivait dans une stratégie de rupture totale avec l'Empire ottoman et son corollaire le monde musulman, jugé rétrograde à l'époque et dans une optique de ralliement du monde occidental, symbole de modernité et de sécularisation.

Pour revenir à ce qui est couramment appelé la "Révolution des signes", nous pouvons dire qu'une langue, censée n'être qu'un moyen de communication, se trouve du jour au lendemain, mêlée à toute une politique d'État. Cela nous amène à avancer aussi que la "révolution" kémaliste, qui devait chambouler toutes les habitudes turques : système politique, organisation sociale, droits de la femme, mode vestimentaire, appel à la prière, l'Occident comme référence socioculturelle, avait besoin d'un moyen à même d'inculquer tous ces changements à la société locale. C'était à la fois un moyen, un symbole de rupture avec les Arabes et d'allégeance aux Occidentaux. Ce qui est intéressant dans l'expérience turque, c'est que les changements politiques peuvent entraîner des changements d'ordre linguistique. Dès lors, la langue devient un instrument de pouvoir destiné à la mobilisation des masses, à la 


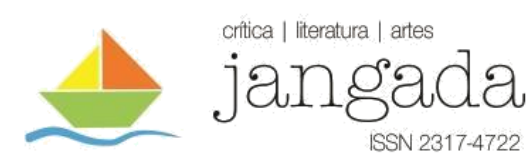

propagande et à d'autres fins que seuls les politiques connaissent. Mais cette vision d'Atatürk nous rappelle l'idée selon laquelle la langue est une superstructure. C'est-à-dire, à un système économique donné correspondrait une langue et tout changement de ce système entrainerait des modifications au niveau de celle-ci. Mais est-ce que ce changement est volontaire ? Ou est-ce une suite logique du bouleversement économique créé par Atatürk ? Nos recherches concernant une éventuelle influence marxiste sur le président turc n'ont pas abouti à quelque chose de pertinent pouvant apporter une réponse claire à notre questionnement. S'il est vrai qu'Atatürk avait de bons rapports avec Lénine, son intérêt pour le marxisme reste à prouver. De par son parcours même, le président turc ne s'est jamais montré marxiste ou adepte des idées de Marx. Donc, nous n'avons pas le choix quant à la justification de la décision d'Atatürk de modifier certains aspects de la langue turque. Nous restons convaincus que le changement linguistique impulsé par ce président était une manière comme une autre de rompre avec le monde musulman et de nouer de nouveaux rapports avec le monde occidental. Mais cela, encore une fois, ne nous empêche pas de dire que le changement linguistique en Turquie est dû à la politique prônée par les réformateurs de ce pays. Dans ce cas, la notion de superstructure ne peut être retenue à partir du moment où celle-ci désigne "dans le vocabulaire marxiste, un système d'institutions, d'idées..., correspondant à une forme déterminée de conscience sociale et dépendant d'une «base », d'une structure économique" (LE PETIT ROBERT, 2014). Cependant, cette idée de superstructure a déjà constitué un sujet de débat très animé en particulier en Russie, pays où les hommes politiques se sont immiscés, à l'instar d'ailleurs d'Atatürk avec sa théorie de langue-soleil, dans les affaires de la linguistique. C'est qu'en définitive, la langue et, par ricochet, la linguistique sont devenues des otages de la politique.

\section{STALINE : LANGUE ET LINGUISTIQUE, UNE AFFAIRE D'ÉTAT}

Une autre leçon sur le rapport langue-politique nous vient cette fois-ci de Russie et grâce, plus particulièrement, à Staline, surnommé le "Petit Père des peuples". Cet inconditionnel du Léninisme succède à Lénine à la tête de L'URSS en 1920. Gérant les affaires de l'Union soviétique d'une main de fer, il a su s'imposer au sein du peuple russe pour se faire aimer, mais pour se faire détester aussi. Tout était sous son contrôle, des plus grandes affaires d'État aux petits problèmes de linguistique. Nous n'allons pas nous attarder sur ses exploits ou ses échecs, ce n'est pas le but de notre travail. Nous allons juste mettre l'accent sur la conception politique 
de la langue chez Staline. Ainsi, après avoir été un fervent défenseur des thèses de Nicolas Marr, célèbre linguiste russe, le Petit Père des peuples s'en démarque en 1950 en tenant les propos suivants : "N. Marr a introduit dans la linguistique la thèse erronée, non marxiste de la langue considérée comme une superstructure, il s'y est empêtré lui-même et y a empêtré la linguistique" (STALINE, 1950). Cette critique met fin à plus de vingt ans de culte du Marrisme et prouve par la même occasion que le pouvoir politique, après avoir vanté les mérites de cette théorie et imposé sa conception au sein de la société russe, se rétracte et déclare non fondée toute une doctrine linguistique. Cela montre que, même en matière de langue, c'est au pouvoir que revenaient le premier et le dernier mot. La langue a donc été une vraie affaire d'État du temps de Staline. Pour approfondir cette question, il est important de reprendre quelques points abordés par ce dernier dans son article publié par le journal La Pravda le 20 juin 1950.

Staline prend donc la parole et traite des problèmes de linguistique. Après avoir lu son article, nous avons jugé opportun de ne reprendre que quatre points. Le premier, et il est très important, concerne l'idée selon laquelle la langue est une superstructure (ce sujet, nous l'avons déjà évoqué dans la partie réservée à la politique linguistique turque). C'est-à-dire, toute base économique, appelée infrastructure, suppose l'existence de superstructures qui lui sont propres. Ces superstructures concernent, entre autres, la politique, le social et la religion. Le plus intéressant dans cette théorie marxiste, c'est qu'à chaque fois qu'il y a une nouvelle infrastructure économique, la superstructure est chamboulée. Examinons ce qu'en dit Staline : "La base est le régime économique de la société à une étape donnée de son développement. La superstructure, ce sont les vues politiques, juridiques, religieuses, artistiques, philosophies de la société et les institutions politiques, juridiques et autres qui leur correspondent” (1950).

Quoi de plus intéressant qu'un marxiste pour expliquer une notion de base relevant de sa doctrine. Donc, d'un côté, il y a la base ou l'infrastructure économique, de l'autre, un ensemble de structures caractérisant la vie sociale : politique, philosophie, idéologie... Pour revenir au sujet, qui nous intéresse et que nous pouvons résumer à travers cette question : la langue est-elle une superstructure ?, nous pouvons dire que la réponse est simple. C'est celle donnée par Staline lui-même dans son article (1950). Pour lui, la langue n'est pas une superstructure dans la mesure où, malgré les changements de la base économique russe, avec la suppression du capitalisme et l'installation du socialisme, la langue n'a pas changé. Il reconnait certes que de nouveaux mots ont fait leur apparition dans la langue russe, mais cela est dû, selon lui, à l'émergence d'une nouvelle doctrine incarnée par le socialisme. En témoigne 
aussi le fait que la majeure partie du vocabulaire et la grammaire sont restées inchangées. Pour le Petit Père des peuples :

La langue [...] diffère radicalement de la superstructure. La langue est engendrée non pas par une telle ou telle base, vieille ou nouvelle, au sein d'une société donnée, mais par toute la marche de l'histoire de la société et de l'histoire des bases au cours des siècles [...] La langue peut servir l'ancien régime agonisant aussi bien que le nouveau régime ascendant, l'ancienne base aussi bien que la nouvelle. (STALINE, 1950)

L'autre raison justifiant le rejet de la thèse de la superstructure linguistique est le caractère éphémère de la superstructure. Celle-ci disparait après la disparition de la base.

Le deuxième point traité par Staline est celui relatif à la thèse marriste selon laquelle il existe des langues de classe. Autrement dit, les classes sociales, nobles, aristocratiques, prolétariennes, riches ou pauvres ont, chacune, une langue qui les distingue les unes des autres. Là encore, rejet total de cette idée. La langue :

Est l'œuvre non pas d'une classe quelconque, mais de toute la société, de toutes les classes de la société [...] Le rôle d'instrument que joue la langue comme moyen de communication entre les hommes ne consiste pas à servir une classe au détriment des autres classes, mais à servir indifféremment toute la société, toutes les classes de la société. (STALINE, 1950)

Classe ou pas, pour Staline, il y a une seule langue, nationale, pour tout le monde. Cette idée de langue de classe est décriée par cet homme politique qui pense que les langues parlées par certaines classes sont des dialectes ou des jargons. C'est d'ailleurs le troisième point que nous allons aborder dans les paragraphes qui suivent. Staline relègue au second plan les dialectes nationaux, il les classe dans la catégorie des jargons. Pour lui, seule la langue nationale unique compte. Les “[...] dialectes et jargons sont souvent qualifiés à tort de langues [...] (Ils) n'ont pas de système grammatical ni de fonds de vocabulaire propres" (1950). Cela montre par ailleurs que Staline, qui n'est pas linguiste de formation, confond les concepts de base de la sociolinguistique. Cela se comprend, ce n'est qu'un politique. Mais le fait de mettre sur le même pied d'égalité jargon et dialecte en dit long sur la témérité de certains hommes politiques qui s'aventurent dans des questions qui ne relèvent pas de leur ressort. En même temps, le problème Jangada | ano 9, nr. 17, jan/jun, 2021 | ISSN 2317-4722 


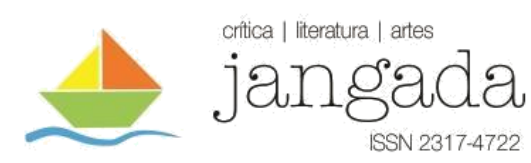

qui se pose est que la langue, étant un instrument de pouvoir, ne peut échapper ni au contrôle de celui-ci ni à son apanage.

Le dernier point relevé dans l'article, cité précédemment, porte sur le caractère social et l'étude diachronique de la langue. À la manière d'un linguiste, Staline affirme que la langue est un fait social par excellence et que son analyse doit être basée sur le paramètre historique :

La langue compte parmi les phénomènes sociaux qui agissent pendant toute la durée de l'existence de la société [...] Pas de langue en dehors de la société. C'est pourquoi l'on ne peut comprendre la langue et les lois de son développement que si l'on étudie la langue en relation étroite avec l'histoire de la société, avec l'histoire du peuple auquel appartient la langue étudiée [...] (1950)

Voilà donc l'apport de la politique stalinienne à la linguistique, apport dont l'enjeu principal reste le pouvoir : celui de faire main basse sur toutes les affaires étatiques, celui d'éviter tous les pluralismes dans la société : linguistique ou autre. Si nous réexaminons les points relatifs à la dichotomie : langue-dialecte, nous allons nous rendre compte que cette idée de langue unique n'est qu'un rempart contre une éventuelle atteinte à l'unité du peuple et par ricochet à la survie du pouvoir en place. Minimiser, dénigrer ou méconnaitre carrément les dialectes participe de la volonté politique d'éviter l'émergence des minorités et la remise en cause du système.

Pour ce qui est de la langue de classe, il n'y en a certainement pas mais il existe des discours de classe. La langue se présente en fait comme un réceptacle dans lequel les différentes classes de la société puisent leurs mots et c'est en fonction de ceux-ci que nous avons affaire à un discours communiste, à un discours islamiste, à un discours démocratique... Une seule langue peut en effet permettre d'avoir plusieurs discours. Le discours c'est la langue en situation, en action. Donc, cette question de langue de classe relève plus du discours que d'autre chose. Ce qu'il faut retenir enfin de ce tableau concernant la Russie, c'est que la linguistique est une affaire éminemment politique.

\section{HITLER : LA LANGUE COMME MOYEN DE PROPAGANDE POLITIQUE}


Adolf Hitler s'installe au pouvoir à partir de 1933. À la fois chancelier du Troisième Reich et guide, le Führer, cette figure emblématique de l'Allemagne nazie va entraîner le monde entier dans une seconde guerre mondiale aux conséquences ravageuses. Ce Führer va mobiliser derrière lui une bonne partie des Allemands. Sorte d'ensorceleur des foules, il avait le verbe et savait exercer une influence remarquable sur son auditoire. Mais cela n'était possible qu'en attribuant à la langue allemande des objectifs autres que ceux pour lesquels elle était employée jusque-là. La nouvelle doctrine politique, prônée par le nazisme, nécessitait le recours à un moyen d'expression à même de rassembler autour d'elle le maximum d'adeptes. C'est pour cette raison que certaines structures, lexicales et syntaxiques en particulier, de la langue allemande ont été modifiées :

La langue nazie $[\ldots]$ change la valeur des mots et leur fréquence, elle transforme en bien général ce qui, jadis, appartenait à un seul individu ou à un groupuscule, elle réquisitionne pour le Parti ce qui, jadis, était le bien général et, ce faisant, elle imprègne les mots et les formes syntaxiques de son poison, elle assujettit la langue à son terrible système, elle gagne avec la langue son moyen de propagande le plus puissant, le plus public et le plus secret. (KLEMPERER, 1996, p. 41)

L'objectif était donc clair, les Nazis, et à leur tête Hitler, voulaient endoctriner leur peuple au moyen de la langue. V. Klemperer avance que cette langue, initialement utilisée par le parti national-socialiste, est devenue, une fois que ce dernier a pris le pouvoir, la langue du peuple. “[...] elle s'empara de tous les domaines de la vie privée et publique : de la politique, de la jurisprudence, de l'économie, de l'art, de la science, de l'école, du sport, de la famille, des jardins d'enfants, et des chambres d'enfants" (1996, p. 45). C'est cela d'ailleurs le but de toute propagande politique : amener le peuple à adhérer à tous les principes voulus et défendus par le pouvoir et quoi de plus efficace que la langue pour réaliser une telle tâche. Mais pour mettre en exergue toutes les facettes de cette langue, nous allons exposer ses caractéristiques une à une.

Commençons par le caractère monotone de la langue nazie et cela n'est pas une exception dans la mesure où toutes les langues à propagande recourent à la répétition de certains mots et expressions. Selon Klemperer (1996, p. 49), les discours nazis étaient truffés de 


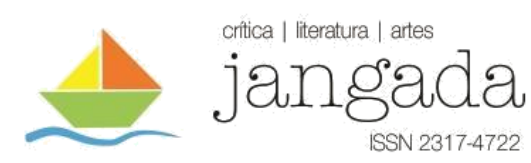

“harangues". C'était une langue pauvre tant ces discours et les différents écrits des responsables nazis se ressemblaient : c'était le même style, les mêmes thèmes abordés.

La deuxième caractéristique de cette langue est qu'elle "tue" l'individu. Elle le vide de sa substance. Il devient une sorte de "tête de bétail, sans pensée ni volonté, dans un troupeau mené dans une certaine direction et traqué [...]" (KLEMPERER, 1996, p. 49). Les mots sont forts mais l'auteur veut apparemment insister sur les effets destructeurs de cette langue dans le sens où la notion d'individu disparait et laisse place à la masse, à la foule. Là encore, l'auteur nous rappelle un des slogans des Nazis “Tu n'es rien, ton peuple est tout” (1996, p. 49). L'Allemand à cette époque n'existait que dans et par le peuple. Tout se ramenait à celui-ci ; l'individu, qui voulait émerger ou prendre des initiatives, n'avait à le faire qu'en pensant au peuple.

La troisième particularité (KLEMPERER, 1996, p. 50), caractérisant cette langue, est son fanatisme. Du moment qu'elle s'adresse à la masse, elle a tendance à inculquer au peuple la fanatisation. Elle fait en sorte que ce dernier devienne sous l'emprise de la doctrine nazie, pas d'acceptation de ce qui sort du cadre nazi d'où son intolérance face à tout ce qui s'oppose à cette doctrine.

Une autre caractéristique de cette langue est le recours quasi systématique au mot peuple chez les partisans du nazisme qui l'employaient abusivement dans des expressions comme : “ «fête du peuple »(Volksfest), « camarade du peuple » (Volksgenosse), « communauté du peuple » (Volksgemeinschaft), «proche du peuple » (volksnah), « étranger au peuple » (volksfremd), « issu du peuple » (volksentstammt...” (KLEMPERER, 1996, p. 58). Tous les discours ou les écrits des nazis à l'époque comportaient des passages où ces expressions étaient légion. C'était une façon aussi de faire sentir au peuple que tout émanait de lui, qu'il était en quelque sorte le vrai détenteur du pouvoir.

Il faut noter aussi que durant le règne du nazisme, beaucoup de sigles, des abréviations en fait, ont fait leur apparition. Klemperer en cite quelques-uns : "BDM (Bund Deutscher Mädel : Ligue des filles allemandes), HJ (Hitler Jugend : Jeunesse hitlérienne), DAF (Deutsche Arbeitsfront : Front du travail allemand " (1996, p. 33). Nous pouvons rajouter à cette liste le sigle "SS" qui renvoie à "Schutzstaffel", échelon de protection en français (LE PETIT ROBERT, 2014).

Il faut signaler également l'emploi de mots nouveaux comme par exemple l'adjectif “combatif" pour faire face à une nouvelle réalité politique où l'esprit allemand doit être mis sur le devant de la scène. Le mot "guerrier", proche sémantiquement, existait bien dans cette langue 


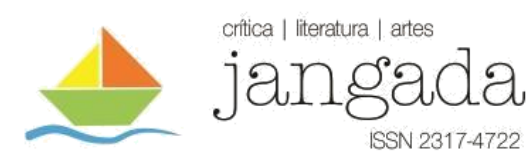

mais les tenants du nazisme, dans leur quête d'une langue à la hauteur de leurs ambitions, se sont rabattus sur “combatif”. Pour Klemperer :

La langue du nazisme a répandu l'usage de « combatif », adjectif nouveau et rare $[\ldots]$ pour en faire un de ses mots favoris. « Guerrier » était trop étroit ; il n'évoquait que les choses de la guerre et c'était aussi un adjectif trop franc qui trahissait l'humeur querelleuse et la soif de conquêtes. Tandis que « combatif »! Cet adjectif désigne d'une manière plus générale une tension de l'âme et de la volonté qui, en toutes circonstances, vise à l'affirmation de soi par l'attaque et la défense, et qui n'est encline à aucun renoncement. (1996, p. 28)

N'est-ce pas là toute la conception du peuple allemand dans la perspective nazie ? Il se trouve que le nazisme a exacerbé le sentiment d'héroïsme chez les Allemands d'où le recours à ce nouveau mot.

Mais le fait le plus marquant de la langue nazie est l'emploi de mots étrangers, notamment français, dans les discours d'Adolf Hitler. Bien que des expressions similaires existent en allemand, le Führer utilisait, par exemple, "Garant" à la place de Bürge, "diffamieren" au lieu de schlechtmachen (KLEMPERER, 1996, p. 33), “diskriminiern" (discrimination) ou encore "defätismus" (défaitisme) (Szambien, 2003, p. 122). Ce choix de mots français était volontaire de la part du Führer. C'était sa stratégie, à lui, pour que son discours ait plus d'effet sur ses auditeurs. Selon Klemperer (1996, p. 33), cela a eu les effets escomptés surtout sur ceux qui ne connaissaient pas ces mots. Tous les moyens deviennent apparemment bons pour asseoir son autorité et faire main basse sur les affaires du peuple.

Nous n'avons pu reprendre tous les points caractérisant la langue nazie dans la mesure où ces quelques éclaircissements suffisent, à notre sens, à lever le voile sur ce qui se cache derrière le rapport du politique au linguistique. De simple moyen de communication, la langue passe à un instrument d'embrigadement des masses. Des mots nouveaux y sont intégrés, des sens inhabituels sont acceptés et, parfois même, des structures syntaxiques sont modifiées rien que pour faire comprendre au peuple le bien-fondé de la doctrine qu'on met devant lui. L'adhésion du peuple est vite acquise vu la force de persuasion dont usent les tenants des obédiences de tout bord. L'illustration de tout cela est donc fournie par le nazisme qui, durant plus de 12 ans de règne, a su, au moyen de la langue, intéresser et subjuguer le peuple et le 


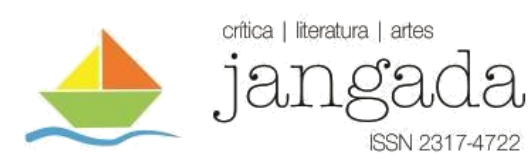

conduire à sa perte. Ce que nous venons d'exposer nous amène à dire que certains évènements politiques font que certaines langues, à un moment donné de leur histoire, sont qualifiées de langues de propagande, de religion, de guerre ou de démocratie. Autrement dit, une langue qui a connu et propagé les idées propagandistes est plus apte à véhiculer cela qu'une langue qui n'a jamais connu d'événements de la sorte. C'est que, pour la première, tout est prêt, le vocabulaire étant déjà formé et utilisé tandis que, pour la deuxième, tout un travail d'appropriation des mots qu'il faut doit être réalisé. C'est le même constat qui s'applique, nous semble-t-il, à la démocratie et aux langues qui sont susceptibles de la véhiculer. Mais ce qui intrigue dans le cas allemand, c'est que cette langue de propagande du temps d'Hitler est celle-là même qu'on emploie de nos jours en Allemagne pour parler de la démocratie.

\section{ITALIE : DE LA POLITIQUE AUTARCIQUE FASCISTE ̀̀ LA LANGUE AUTARCIQUE}

Dans cette partie, nous allons voir comment une politique étatique a eu un impact considérable sur la langue italienne et ce, depuis l'apparition du fascisme. Système politique basé sur le totalitarisme, le fascisme va être appliqué et imposé aux Italiens, de 1922 à 1943, par le biais de Mussolini. Fervent défenseur du tout national, celui-ci choisit, quelques années après la prise du pouvoir, la voie de l'autarcie pour l'Italie. Désormais, les importations se font au comptegouttes et les Italiens doivent vivre de leur production locale. "Mussolini veut en effet que l'Italie se suffise à elle-même en matière agricole. Il lance en grande pompe la «bataille du blé » en 1925" (LARANÉ, 2013). Mais ce n'est pas qu'en matière agricole que cette autosuffisance devait se concrétiser, la langue aussi devait se suffire à elle-même et éviter l'emprunt aux langues étrangères. Mais dans le cas linguistique, le pouvoir de Mussolini semble avoir procédé par étapes. Selon Gabriella B. Klein (2004, p. 93), le paysage linguistique italien, durant la période fasciste, a connu trois phases : une phase, se situant dans les années 1920, où le pouvoir en place a œuvré pour l'unification de la langue italienne au détriment des dialectes locaux ; une deuxième phase, située dans les années 1930, où l'on a cherché à appliquer le principe de l'unité linguistique égale unité politique et donc celle du peuple ; une troisième phase enfin, à la fin des années 1930, où s'est affirmée la langue autarcique, synonyme du refus de tout ce qui était étranger. Domineco D’Oria donne plus de détails en ce qui concerne la troisième phase. D'après elle : 
C'est en 1937 que la politique fasciste de répression des mots étrangers se définit comme politique linguistique et politique antibourgeoise. Le climat idéologique et de guerre, alimenté par la politique de propagande fasciste, avait inculqué chez les Italiens un sentiment de xénophobie. (1985)

Cette mesure avait donc un double objectif : la suppression des mots étrangers présents dans la langue italienne et l'élimination en quelque sorte de la classe bourgeoise.

Deux faits importants caractérisent par ailleurs cette décision d'italianiser la langue locale et de l'épurer de tous les termes d'origine étrangère : le premier concerne la promulgation d'une loi en décembre 1940 interdisant aux Italiens le recours aux mots autres qu'italiens. Le deuxième fait porte sur la sortie en 1941 du livre (tome1) : Vocabolario della lingua italiana (Vocabulaire de la langue italienne) (D’ORIA, 1985).

\section{COMMENT S'EST DÉROULÉE L'APPLICATION DE CETTE POLITIQUE LINGUISTIQUE AUTARCIQUE?}

Là encore, les fascistes semblent avoir pris tout leur temps pour imposer l'autarcie en matière de langue. Leur stratégie était simple : il s'agissait d'abord d'appliquer cette mesure en douceur, discrètement, quitte à ne toucher qu'une partie infime de la population locale :

La politique fasciste dans le domaine linguistique s'est exercée non pas directement sur tous les Italiens, mais à travers des ordres donnés à la presse [...] et par des campagnes contre l'introduction des mots étrangers pour imposer une autarcie linguistique, considérée comme une nécessité permanente, et non pas comme une exigence du moment. (D’ORIA, 1985).

Les lois promulguées en matière de langue n'étaient donc pas destinées à l'ensemble des Italiens mais à une partie d'eux dans la mesure où la majorité recourait au dialecte sur lequel le pouvoir continuait à fermer les yeux. De plus, ces lois visaient plus l'écrit que l'oral (D’ORIA, 1985). Mais les fascistes étaient de fins stratèges dans ce domaine. Ainsi, selon Domenica Doria (1985), malgré le caractère restreint des textes de lois, restreint dans le sens où ils ne s'adressaient pas à tous les Italiens, force est de constater que l'interdiction des mots 
étrangers, dénommés "forestierismi et esotismi”, a touché progressivement la majorité par le biais notamment de l'école et des dictionnaires qui se sont révélés de redoutables moyens pour répandre la propagande fasciste.

\section{COMMENT CES DICTIONNAIRES SE PRÉSENTAIENT-ILS ?}

Les dictionnaires, édités à l'époque, étaient soit terminologiques, soit des dictionnaires de langue. Les premiers avaient pour objectif la suppression des emprunts relatifs à tous les mots techniques et scientifiques et leur remplacement par des termes italiens. Les seconds se présentaient, pratiquement, de la même façon mais ils portaient sur la langue de tous les jours (D'ORIA, 1985).

Il faut noter que ces dictionnaires "se caractérisent par un langage politique marqué qui transgresse toutes les stratégies de neutralité et de discrétion, et, par-là, rejoignent les dictionnaires d'interdiction et de prescription et donc les textes législatifs du régime" (D'ORIA, 1985).

Une autre caractéristique de ces dictionnaires est l'usage d'une langue militaire dans la mesure où, selon l'auteur cité précédemment, ils ne justifient nullement les interdictions qu'ils comportent. Ainsi, le ton est proche de celui des militaires, péremptoire, arbitraire. Le lecteur n'avait qu'à s'y soumettre et c'est tout. Aucune explication ne lui était fournie. C'était des dictionnaires prescriptifs, rien de plus.

\section{COMMENT LES LEXICOLOGUES DE L'ÉPOQUE FASCISTE PROCÉDAIENT-ILS POUR CONCRÉTISER CETTE AUTARCIE?}

Ils procédaient de manière tout à fait simple. En fait, ils suivaient à la lettre les recommandations de la commission ad hoc, installée en 1924 par la société Dante Alighieri et qui était chargée de proposer des mots italiens à employer à la place de ceux de l'étranger. Quand les mots italiens n'étaient pas disponibles, il fallait les puiser dans la langue italienne archaïque ou dans le latin. Ce "procédé [...] sera largement utilisé par le dictionnaire de l'Accademia d'Italia qui aura recours souvent à l'étymologie latine pour défendre-imposer le mot italien équivalent" (D’ORIA, 1985). Cela montre à quel point les mots étrangers étaient honnis par les fascistes. 
Mais à quoi tout cela rimait-il ? Pourquoi cette haine des mots étrangers ? Cela avait-il une raison autre que linguistique?

\section{LES RAISONS QUI SE CACHENT DERRIÈRE LA LANGUE AUTARCIQUE}

Il ne faut pas chercher ces raisons en dehors du cadre politique. Le refus des mots étrangers relève du refus même de l'étranger et en particulier de la France, considérée comme le pire ennemi des fascistes. Selon Domenico D’Oria :

L'ennemi principal, vers 1940, était la France, et c'était donc la langue française qu'il fallait combattre par tous les moyens, comme le montrent les nombreux ordres à la presse. La lutte contre les mots étrangers, au nom d'une autarcie linguistique, n'était menée que contre les mots français [...] Ce qui laisse penser que derrière l'étiquette d' « autarcie linguistique » se cache le vrai problème de la lutte contre une langue et une seule. (1985)

Mais cela répond à toute une politique, celle du fascisme et celle de tous les totalitarismes dont le credo est la conservation du pouvoir par tous les moyens, y compris le moyen linguistique.

En revanche, ce qui attire l'attention dans cette expérience fasciste, c'est que le principe appliqué à la langue italienne émane de l'économie. L'autarcie, nous l'avons vu plus haut, a d'abord été appliquée en matière d'agriculture puis elle a été transposée dans le domaine de la langue. Est-ce que cela nous permet de dire que la langue fasciste est une superstructure ? Nous sommes tenté de l'affirmer mais vu que l'idée même d'autarcie vient de la doctrine politique fasciste, notre tentation s'arrête net et les faits nous renvoient toujours au politique, raison pour laquelle nous ne pouvons que retenir le fait que la langue sert parfois d'adjuvant de taille pour asseoir ou imposer un système politique.

\section{LA GRÈCE : LANGUE DE LA DICTATURE VS LANGUE DE LA DÉMOCRATIE}

La Grèce mérite amplement sa place dans cet article dans le sens où elle va nous servir d'exemple pour illustrer le cas des langues qui s'affrontent ou plus simplement le cas de la langue du pouvoir et de la langue du peuple. Nous allons voir en effet comment un idiome, 


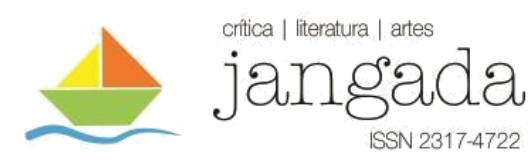

longtemps imposé par une frange détenant le pouvoir, va céder face à la détermination du peuple.

Cet État européen est souvent cité comme un exemple typique de diglossie qui désigne, nous devons le rappeler, une situation linguistique où deux langues en usage dans un pays donné sont utilisées inégalement selon le contexte de la communication. L'une, appelée "variété haute", est réservée au formel : discours politique, prêche religieux, discours didactique... L'autre, dénommée "variété basse", est plutôt destinée au non officiel : réunion de famille, relations amicales... La diglossie semble être présente un peu partout dans le monde. Il suffit juste de s'intéresser à l'emploi des langues dans certains pays pour s'en faire une idée.

Le cas grec, qui s'étale sur tout le $\mathrm{XX}^{\mathrm{e}}$ siècle, est qualifié de diglossique dans la mesure où il y avait deux langues en concurrence : la katharévoussa, langue officielle et la démotiki, langue populaire.

\section{MAIS QU'EST-CE QU'AU JUSTE LA KATHARÉVOUSSA ET LA DÉMOTIKI ?}

L'explication que nous avons fournie plus haut ne rend pas compte de tous les aspects de ces deux langues. C'est pour cette raison que nous avons jugé utile de reprendre les deux définitions données par Georges Prévélakis. Selon ce spécialiste de géopolitique, la katharévoussa, langue inventée par Koraïs, est "une version purifiée de la langue grecque, présentée comme débarrassée des scories accumulées par l'histoire et le contact avec d'autres peuples" et la démotiki est une "langue aussi artificielle que la katharévoussa, puisque ses règles ont été élaborées par des linguistes, son vocabulaire était plus proche de celui des parlers locaux, et ses règles moins strictes" (2005, p. 47).

Cette précision nous permet de distinguer d'un côté, une langue proche du grec classique mais épurée des relents historiques et de l'influence d'autres cultures et là, l'auteur voulait sûrement parler des emprunts à d'autres langues. Cela nous rappelle les efforts déployés par les fascistes italiens pour débarrasser leur langue des mots étrangers. Cela veut dire aussi que cette langue était celle du pouvoir en place puisque seul ce dernier pouvait décréter une telle qualité linguistique. D'un autre côté, cette précision nous amène à dire que la démotiki présente des similitudes formelles avec la précédente. La seule différence de celle-ci par rapport à celle-là est qu'elle émane des dialectes locaux, donc c'était la langue du peuple. 


\section{LA KATHARÉVOUSSA ET LA DÉMOTIKI : HISTOIRE D’UN CONFLIT}

Selon Louis-Jean Calvet (2016, p. 250), les problèmes linguistiques grecs ont commencé en novembre 1901. Ainsi, suite à la sortie d'un exemplaire de la Bible traduite en démotiki, des émeutes ont éclaté dans de nombreux endroits en Grèce. Les manifestants avaient un seul mot d'ordre : les traducteurs de la Bible devaient être excommuniés. En effet, avant cet événement, la traduction se faisait uniquement en katharévoussa et ce, à partir du grec classique. La démotiki, elle, était une langue minorée, jugée comme étant un idiome vulgaire. C'est pour cette raison d'ailleurs qu'il y a eu une levée de boucliers de la part de certains Grecs qui avaient considéré la traduction en démotiki comme un sacrilège. Des mesures ont été alors prises par la haute autorité de l'Eglise grecque qui a formellement interdit de vendre les exemplaires en démotiki et à tous ceux qui ne s'y soumettraient pas, elle promettait l'excommunication. C'est donc la katharévoussa qui va continuer à être employée comme langue étatique et ce, jusqu'à 1974, année durant laquelle un nouveau pouvoir met fin à la dictature qui sévissait jusque-là. Deux années plus tard, la démotiki est introduite dans l'enseignement et utilisée dans l'administration. Cette langue, synonyme de la disparition de la dictature et représentant le pouvoir du peuple, va petit à petit remplacer la katharévoussa en subissant année après année des aménagements. "On simplifie le système des accents, puis le lexique sera modifié, avec le souci constant de rapprocher la langue écrite de la langue parlée [...]" (CALVET, 2016, p. 252).

\section{LA FACE CACHÉE DU CONFLIT LINGUISTIQUE GREC}

Le conflit linguistique grec était éminemment politique. La vraie raison est à chercher en effet dans le paysage politique grec du $\mathrm{XX}^{\mathrm{e}}$ siècle durant lequel il y a avait d'un côté, les dictateurs, détenteurs du pouvoir et favorables au maintien de la katharévoussa. Ils avaient à leur disposition les moyens de l'État et ils avaient donc toutes les facilités pour promouvoir et surtout imposer leur langue. De l'autre, il y avait les défenseurs de la démotiki, hostiles au pouvoir et à sa politique qui faisait fi des aspirations du peuple. Calvet, de son côté, a analysé la situation en ces termes : “[...] les «belligérants » se divisaient en deux groupes que l'on peut grossièrement définir comme l'un de "gauche", partisan de la démotiki, et l'autre de « droite nationaliste », lié aux intérêts de la religion et défendant le maintien de la katharévoussa" (2016, p. 252). Le problème relève donc, comme nous l'avons précisé au début 
de ce passage, de la vision politique qu'avaient les uns et les autres de la Grèce et cela s'est manifesté à travers un conflit linguistique aux conséquences parfois dramatiques puisque les émeutes de novembre 1901 avaient même fait des morts à cause des langues. Ce qui a d'ailleurs beaucoup intrigué Calvet qui avait du mal à accepter le fait qu'on tue pour des langues. Mais c'était plutôt des tueries « politiques ».

Par ailleurs, Gorges Prévélakis abonde pratiquement dans le même sens mais il apporte un autre éclairage en ce qui concerne ce conflit. Il dit :

La lutte entre les katharévoussiani (adeptes de la katharévoussa, associés aux partis politiques conservateurs) et les démotikistès (ceux qui soutenaient la démotiki, libéraux et communistes) a marqué la vie intellectuelle grecque du $\mathrm{XX}^{\mathrm{e}}$ siècle. Elle s'est terminée par le triomphe de la démotiki en 1976, peu après la fin de la dictature militaire [...] (2005, p. 47)

Donc, ce conflit linguistique est beaucoup plus profond que cela dans la mesure où il renvoie à toute la "vie intellectuelle" de la Grèce à un moment donné de son histoire.

Mais, ce qui nous intéresse dans tout cela, c'est le rapport langue-politique. Il s'avère en fait que la katharévoussa était une langue de dictature, incarnant le despotisme, l'autorité débridée de l'État et que la démotiki était une langue de la démocratie, symbolisant l'accès du peuple grec au pouvoir. Dans ce cas, nous ne pouvons évidemment pas parler de superstructure mais de la qualité de la langue, elle-même, celle qui fait que telle langue se prête mieux à l'exercice de la démocratie parce qu'elle émane du peuple. C'est sa langue, c'est celle qu'il comprend et qu'il pratique dans sa vie de tous les jours. C'est celle qui l'unit et c'est celle aussi au moyen de laquelle il peut apporter le changement politique voulu. Ce cas nous amène à conclure que certains bouleversements politiques ont besoin non pas de modifier la langue en termes de structures lexicales ou autres, mais d'une langue propre, susceptible d'effacer une fois pour toutes tous les relents du système déchu et susceptible de montrer que c'est au peuple que revient le dernier mot. Le cas grec est, en définitive, un exemple du combat que peuvent se livrer deux langues représentant deux politiques diamétralement opposées.

\section{CONCLUSION}


Ce qui revient le plus dans les cas examinés ci-dessus, c'est le fait que chaque fois qu'un changement politique a lieu, un changement linguistique se produit et ce, selon la nature du pouvoir qui le provoque. Constituant une partie intégrante de toute politique, la langue s'impose donc comme corollaire de cette dernière. Indispensable aussi bien pour les régimes totalitaires que pour les systèmes démocratiques, la langue est entraînée malgré elle dans les méandres du monde politique qui a toujours besoin d'un moyen d'expression pour exister. Mais ce qui retient l'attention, dans ce que nous avons vu, c'est la primauté du politique sur le linguistique. Nous avons l'impression en effet que tout fait linguistique est tributaire du pouvoir politique. Que ce soit en Turquie, en Russie, en Allemagne ou en Italie, le pouvoir semble avoir fait main basse sur le facteur linguistique pour asseoir son autorité et assurer sa pérennité. Le seul cas où la langue semble avoir eu droit de cité en dehors du cercle du pouvoir, c'est le cas grec où la langue du peuple a été hissée au rang de langue d'État. Là encore, c'est suite à un changement politique que cela a eu lieu. La politique est décidément une condition sine qua non pour l'existence d'une langue qui ne fait en fin de compte qu'exprimer l'idéologie réclamée par cette politique. Cela nous conduit à un autre constat selon lequel une politique basée sur la démocratie ou l'antidémocratie ne peut qu'aboutir à une langue de démocratie ou d'antidémocratie. La langue est ce que la politique ou le pouvoir veut faire d'elle.

\section{RÉFÉRENCES BIBLIOGRAPHIQUES}

AKINCI, M.-A. La réforme de l'écriture turque, 2005. Disponible à : $<$ http://www.bleublancturc.com/Franco-Turcs/MehmetAli/Reforme ecriture turque.pdf >. Accès à : 12, décembre, 2015.

CALVET, L.-J. La Méditerranée. Mer des langues. Paris : CNRS Editions, 2016.

D'ORIA, D. Fascisme et autarcie linguistique, 1985. Disponible à : $<$ http://www.persee.fr/docAsPDF/mots_0243-6450_1985_num_11_1_1204.pdf $>$. Accès à : 03, août, 2017.

GENÇ, O. La Révolution des signes d'Atatürk n'avait qu'un but : réduire l'influence de l'islam, 2016. Disponible à : <chroniquesdegencosman.blogspot.com/2016/02/la-revolution-dessignes-dataturk.html > . Accès à : 20, juin, 2016.

GOUJON, A. Bilinguisme et populisme en Biélorussie. In : Cahiers de l'ILSL. Lausanne, $\mathrm{n}^{\circ}$ 17, p. 45-62, 2004.

KLEIN, G. B. De la langue unitaire à la langue autarcique : le discours sur la langue pendant le fascisme en Italie. In : Cahiers de l'ILSL. Lausanne, n¹7, p. 93-100, 2004.

KLEMPERER, V. LTI, la langue du IIIe Reich, Carnets d'un philologue (Traduit de l'allemand par Elisabeth Guillot). Paris : Albin Michel, 1996.

Jangada | ano 9, nr. 17, jan/jun, 2021 | ISSN 2317-4722

443 | Pá g in a 
LARANÉ, A. 1922 à 1943 : L'État fasciste, 2013. Disponible à : <https://www.herodote.net/1922_a_1943-synthese-101.php >Accès à : 03, août, 2017.

LE PETIT ROBERT, 2014 (version électronique).

MILNER, J.-C. L'amour de la langue. Paris : Le Seuil, 1978.

PRÉVÉLAKIS, G. Géopolitique de la Grèce. Bruxelles : Éditions Complexe, 1997.

STALINE, J. Le marxisme et les problèmes de linguistique. In : La Pravda. Moscou, 20 juin 1950.

SZAMBIEN, W. Berlin, une ville en suspens. Paris : Norma, 2003.

VARDAR, B. Atatürk et la rénovation de la langue turque. In : Dilbilim. Istanbul, n ${ }^{\circ}$ VI, p. 15$22,1981$. 